\section{Perception and memory loss of item and order information in short-term memory*†}

\author{
LESLIE HENDERSON and MICHAEL L. MATTHEWS \\ University of Guelph, Guelph, Ontario, Canada
}

Recognition probes given before or after a series of letters presented at varying rates were used to evaluate perception and memory loss of order and item information. For both order and item information pre- and postprobe functions converged at fast rates of presentation. Performance decrement at fast rates is attributable to perceptual factors and is greater for order than for item information.

Recent evidence indicates that in short-term memory tasks the impairment in performance which occurs with fast sequential presentation is partly attributable to failure of stimulus registration. Thus, Aaronson (1967) has argued that some order errors in short-term memory are due to failure to discriminate successiveness. Scarborough and Stemberg (1964) have shown that perception of the serial order of two items can be markedly impaired even at presentation rates that permit recognition of the items, and foreknowledge of the items does not reduce this impairment of order recognition. More generally, Norman (1966) has claimed that rate of presentation affects the acquisition of items into memory rather than their retention. Matthews and Henderson (1970) have suggested that such perceptual loss may obscure the much-debated relationship between retention and presentation rate. They suggested, in particular, that perceptual loss at fast rates would tend to counteract any improvement in retention resulting from the operation of a time-dependent decay process.

The study reported here was an attempt to separate the effects of presentation rate on perception and on retention of item and order information. In addition, an attempt was made to evaluate the contribution of sequential masking to perceptual loss.

It is, in principle, impossible to construct a memory-free perception task or the converse. One possible strategy is to minimize one of the two components. In the present study a two-stage strategy was used. First, a measure of perceptual loss was achieved by using a task of minimal memory load. Second, by making an

* This paper was presented in part to a meeting of the Experimental Psychology Socicty, London, January 1970.

†DRET Rescarch Paper No. 765. This research was supported by a grant to M. L. Mathews from the Defence Research Board of Canada. The major part of this research was conducted at the Defence Research Establishment Toronto. subtracted from the total loss produced by adding memory load, to obtain a separate estimate of memory loss.

There are two current methods of attempting to minimize memory load. The first involves shortening the series of items for recall, as Scarborough and Sternberg (1964) and Yntema, Wosencraft, and Klem (1964) have done. This method involves the assumption, however, that perceptual difficulty is independent of series length. This assumption seems implausible, especially if, as Aaronson (1968) suggests, perceptual lag accumulates throughout a series.

The other method involves inserting specific test items (probes) before a series begins. This technique has been employed by Aaronson (1968), Eriksen and Spencer (1969), and Henderson and Matthews (1969). Essentially, it involves a visual search process in which the only load on memory is the probes. Perceptual and memory tests, therefore, involve a symmetrical situation with the probes inserted before (preprobes) or after (postprobes) a series, respectively. The additivity assumption, which requires the supposition that the memory load of the preprobes is negligible, is that memory load for the series is added to the perceptual load, which remains constant.

\section{METHOD}

The task material consisted of sequences of eight letters drawn at random without replacement from a 20-letter alphabet, consisting of the consonants minus "W." Presentation was by 8 -mm movie film, 24 frames per second, with one frame per letter. The number of blank frames between letters was varied to yield presentation rates of $1 / 2,2,6$, and 12 items per second.

There were four experimental conditions. Two of these, which were display conditions, were coincident (C) and spaced (S). In Condition C the letters filled the frame and were displayed successively at the same locus. In Condition $S$ the additivity assumption, perceptual loss was items, which were smaller, were sequentially spaced so that each item occurred to the right of the previous one, beginning at the extreme left of the frame and terminating at the right. A red warning signal appeared on the screen for $1 \mathrm{sec}$ immediately before the presentation of each letter sequence. Rates of presentation were block randomized.

The other two conditions were item (I) and order $(0)$ conditions. These involved, respectively, a judgment as to whether each of two probes was present in the series and judgment of the order in which they occurred. Condition I involved a two-bit written response with alternatives, " $Y Y$, YN, NY, NN," arranged equiprobably. Condition $\mathrm{O}$ involved a one-bit response as to whether two adjacent items in the series occurred in the order given by the probes or in the reverse order. These two probe-order conditions were arranged to be equiprobable. The first and last positions in the series were not probed since order judgments involve less processing at these loci, and the recognition of a single item is sufficient for a decision, as is reflected in the data of Donaldson and Glathe (1969). The other positions were probed with equal frequency in random order. The identity of the probes was given auditorily $2 \mathrm{sec}$ before or $0.5 \mathrm{sec}$ after a series.

Twenty Ss performed each of the conditions in a within-Ss design. Ten Ss were paid volunteers from an undergraduate class, and 10 were housewives from the $S$ pool of the Defence Research Establishment Toronto. Ss were tested in groups of 10 .

\section{RESULTS}

The percentages of correct judgments are displayed in Fig. 1. Data points are based on 200 observations. Pre- and postprobe results are displayed together for each of the four conditions, IS, OS, IC, and $O C$. The form of the functions is remarkably similar under all conditions. Preprobe performance remains about $100 \%$ up to 2 items per second and deteriorates in a fairly linear manner between 2 and 12 items per second. Postprobe performance also deteriorates with increased rate of presentation, but the slope is very slight so that the two functions show marked convergence as presentation rate increases. This convergence is limited from $1 / 2$ to 2 items per second by the approximation of preprobe performance to $100 \%$. It is not, however, an artifact of a lower asymptote on postprobe performance, which remains well above the $50 \%$ chance level at 12 items per second. Standard deviations were similar for all conditions, averaging about $6 \%$. 


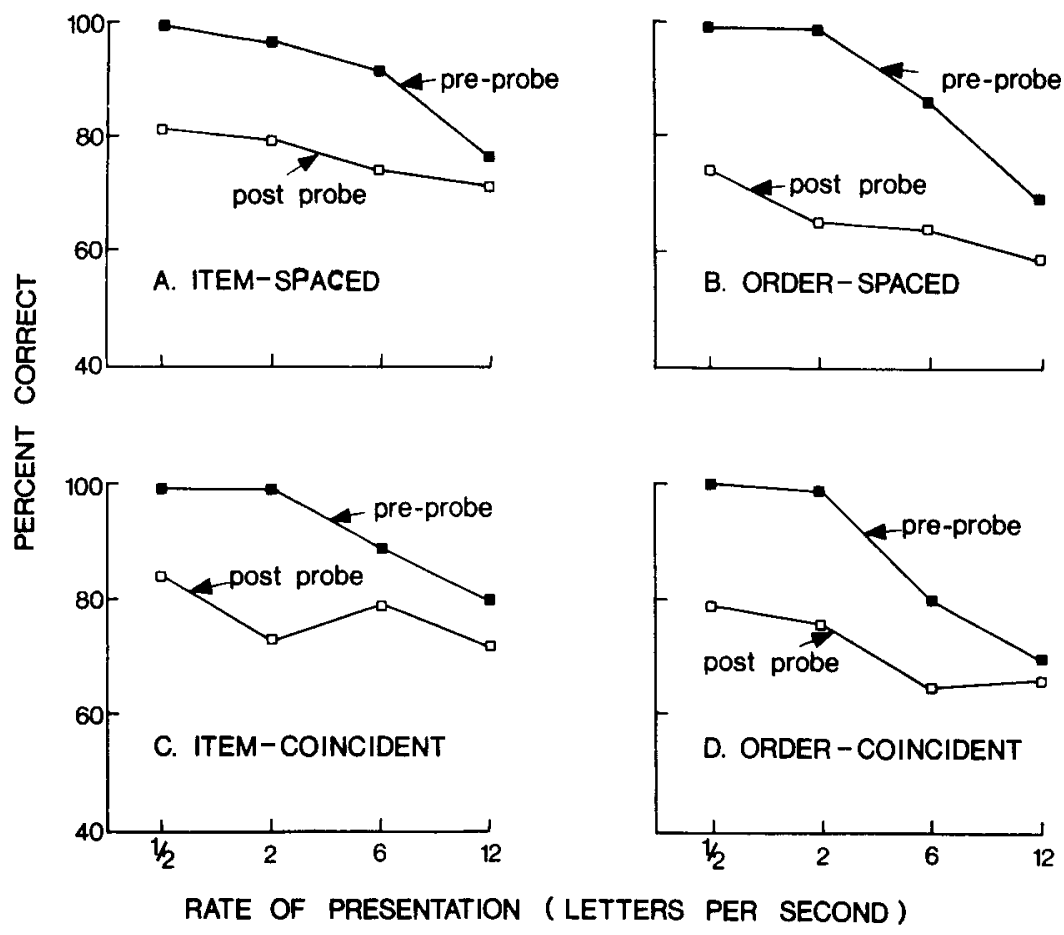

Fig. 1. Preprobe (solid square) and postprobe (open square) performance as a fucntion of presentation rate, for four conditions.

The implications of this convergence when combined with the additivity assumption are apparent in Fig. 2, in which a "purified" retention function has been obtained by subtracting preprobe loss from postprobe loss.

\section{DISCUSSION}

The improvement from 2 to 12 items per second (Fig. 2) is most easily reconciled with the classical notion of a time-dependent decay process, but the absence of systematic changes in a recency effect (from a serial position analysis made of the data) fails to support this position. The upturn at $1 / 2$ item per second may be due simply to the asymptote of preprobe performance or, alternatively, may have substantive implications in terms of the rehearsal which is undoubtedly involved at that rate.

In view of the striking nature of the results the additivity assumption yields, the assumption merits further examination. Certain problems become immediately evident. First, the correlation of Ss' preprobe and postprobe performance was low and nonsignificant with respect to both overall scores and performance at the two fastest rates. Yet the additivity assumption implies a substantial correlation, especially at the faster rates where most of the loss is perceptual, and correlation should therefore be present in both cases.
A second difficulty of the additivity hypothesis is the dependency of the results upon the number of probes used. In the present study, two-item probes were used since, whereas this does not minimize memory load in the preprobe condition, it does permit comparison of item and order performance, both of which demand recognition of two items. In a previous study (Henderson \& Matthews, 1969) using single-item probes but otherwise comparable conditions, preprobe performance deteriorated much less with increased rate, remaining above $90 \%$ at 12 items per second. Any convergence of preand postprobe functions was therefore negligible. Though this lack of convergence can be explained as an artifact of the approximation of preprobe performance to a $100 \%$ asymptote, it suggests that the search for two items used as probes is a complex perceptual task.

A third difficulty for the present test of the additivity hypothesis involves the equivalence of the perceptual demands made by the pre- and postprobe conditions. For the preprobe condition the perceptual process may involve a visual template match, since Posner et al (1969) has shown that an auditory input may be translated into a visual template. However, in the postprobe condition the accumulation of the series in memory may lead to decay of or interference among the visual templates, resulting in the conversion of the task to a name-matching process.
Finally, the additivity assumption implies that perception and retention are successive and independent operations: the "purified" retention measure requires that this be so. There are two ways in which this assumption might be violated. First, it could be considered that in the preprobe condition at fast rates a $S$ allows the material to accumulate in iconic storage and later conducts a memory search. The condition is thus effectively translated into a postprobe one. The better performance with preprobe testing could then be attributed to the processing of earlier items before perceptual lag forced the accumulation strategy to take over.

Though this strategy is logically feasible, there are reasons for excluding it. The virtually perfect performance at very much faster rates obtained by Eriksen and Spencer (1969) argues against the necessity for such a strategy. Furthermore, the successive masking involved in the coincident condition would violate conventional assumptions about iconic storage. In addition, the hypothesized successive and independent nature of the operations is contested by Aaronson's (1968) claim that instructions for subsequent recall alter preprobe performance. Since Aaronson's measure of preprobe performance was response latency, however, it does not necessarily apply; indeed, it would be surprising if such an addition to the response demands did not increase latencies.

While such a possibility is purely speculative, it would result in a difference in the perceptual task in terms of the type of matching operation from pre- to postprobe conditions.

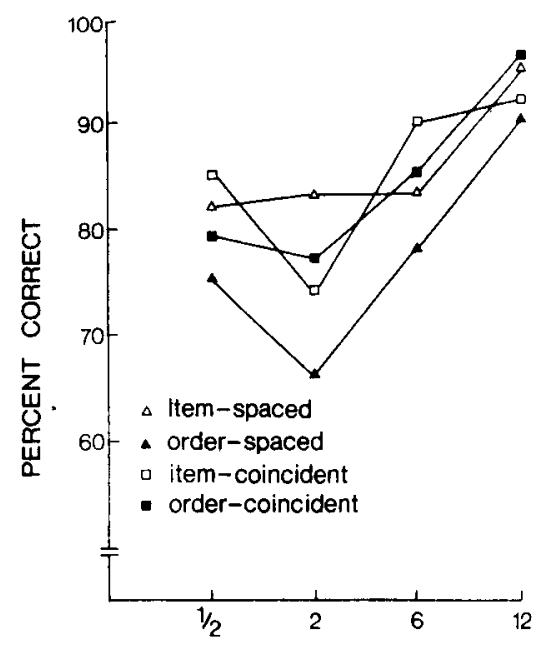

LETTERS PER SECOND

Fig. 2. Derived retention functions on the additivity hypothesis. Preprobe loss has been subtracted from postprobe. 
Concerning the conditions effects, it was found that the spaced material did not result in any systematic superiority over the coincident in preprobe performance. $O S$ is better than $O C$ at 6 items per second and worse at 12, and the same holds for IS and IC. As the size of the spaced items was smaller than that of the coincident, and retinal angle varied according to the S's seating position in the group-testing situation, this absence of any difference cannot be taken as psychophysical evidence bearing directly upon masking factors. It may, however, be the case that any gains from spacing the material are lost by the necessity of making eye movements as the display is presented.

Comparison of preprobe item performance with order reveals the anticipated superiority in the perception of item information. Item perception was clearly superior to order perception with the spaced material (at 6 per second, $\mathrm{t}=3.82, \mathrm{p}<.01$; at 12 per second, $t=4.09, p<.001)$. The superiority of item perception with the coincident material was less marked (at 6 per second, $t=2.91$, $p<.05$; at 12 per second, $t=1.97$, n.s.). This superiority of item perception is to be expected from the results reported by Aaronson (1968) and Scarborough and Sternberg (1964). The fact that the difference was more marked for the spaced material does not confirm the hypothesis that order information should be more readily processed with spaced material because of the redundancy introduced by ordering in both space and time.

A final point also merits attention. As can be seen from Fig. 1, postprobe performance in Condition IC was atypically depressed at 2 items per second. It seems unlikely that this effect is an artifact since two previous experiments (Henderson \& Matthews, 1969) found this depression at 2 per second with IC material, using a single postprobe. At present we are unable to account for this result. The assumption that it represents an unsuccessful changeover from a rehearsal strategy to a passive receiving strategy at this rate is not confirmed by the absence of the effect with spaced material.

\section{CONCLUSION}

The problem of evaluating the perceptual component in immediate recall tasks using fast presentation rates hinges upon two problems; the design of a perceptual test with a negligible memory component and the partitioning problem which is exemplified by the additivity assumption. The preprobe technique seems an adequate solution to the former problem though the multiple-item probe may introduce further complexities. The status of the additivity assumption is rather less certain and so, consequently, is the status of the derived retention functions in Fig. 2. Regardless of whether or not the assumption and its derivations hold however, the finding that at 12 items per second about $90 \%$ of the postprobe loss was obtained with preprobes must enter into any evaluation of the probe-recognition measure of short-term memory.
REFERENCES

AARONSON, D. Temporal factors in perception and short term memory. Psychological Bulle tin, 1967, 67, 130-144.

AARONSON, D. Temporal course of perception in an immediate recall task. Journal of Experimental Psychology, 1968, 76, 129-140. DONALDSON, W., \& GLATHE, H. Recognition memory for item and order information. Journal of Experimental Psychology, 1969, $82,557-560$.

ERIKSEN, C. W., \& SPENCER, T. Rate of information processing in visual perception: Some results and methodological considerations. Journal of Experimental Psychology Monograph, 1969, 79, 1-16.

HENDERSON, L., \& MATTHEWS, M. L. Short term memory at high rates of visual presentation. Paper presented to Psychonomic Society, St. Louis, 1969.

MATTHEWS, M. L., \& HENDERSON, L. Fast presentation rates and the recall of item and order information. Nature, 1970, 226, 374-376.

NORMAN, D. A. Acquisition and retertion in short term memory. Journal of Experimental Psychology, 1966, 72, 369-381.

POSNER, M. 1., BOIES, S, J., EJCHELMAN, W. H., \& TAYLOR, R. L. Retention of visual and name codes of single letters. Journal of Lxperimental Psychology Monograph, 1969, $79,1-16$.

SCARBOROUGH, D. L., \& STERNBERG, S Processing jtems and their order in sequential visual displays. Paper presented to Eastern Psychological Association, Boston, 1967.

SPERLING, G., \& SPEELMAN, R. G. Acoustic similarity and auditory short term memory: Experiments and a model. In D. A. Norman (Ed.), Models of human memory. New York: Academic Press, 1970. Pp. 151-202.

YNTEMA, D. B., WOZENCRAFT, F. T., \& KLEM, L. Immediate serial recall of digits presented at very high rates. Papes presented to Psyshonomic Society, Niagara Falls, 1964.

(Accepted for publication July 20, 1970.) 